\section{Einfluß von Formaldehyd auf die Bindefestigkeit von Sperrholz mit Diisocyanat (PMDI) und Fichten- bzw. Kiefernrindenextrakten als Bindemittel}

B. Dix, Fraunhofer-Institut für Holzforschung, Bienroder Weg 54E, 3300 Braunschweig

Subject: Strenght of plywood bonded with diisocyanate (PMDI) and spruce or pine bark extracts in dependence on formaldehyde.

Material und Methode: Dreilagiges Buchenfurniersperrholz (Furnier: $100 \times 85 \times 1,5 \mathrm{~mm}^{3}$ ) wurde mit Kombinationen aus 7 Teilen PMDI und 3 Teilen $40 \%$ iger Rindenextraktlösung als Bindemittel ohne und mit Zusatz von Paraformaldehyd hergestellt. Leimauftrag beidseits mit $210 \mathrm{~g} / \mathrm{m}^{2}$ je Furnierseite, geschlossene Wartezeit 5..10 min, Preßdruck $1,5 \mathrm{~N} / \mathrm{mm}^{2}$, Preßzeit $6 \mathrm{~min}$, Preßtemperatur $140^{\circ} \mathrm{C}$. Die Bindefestigkeit nach 3 Tagen Normalklima-Lagerung (20/65) (DIN 53255, Lagerung Nr. 1) oder 24 std Kaltwasserlagerung (DIN 53255 , Lagerung Nr. 2) wurde im Zugversuch geprüft.

\begin{tabular}{|c|c|c|c|}
\hline \multirow{2}{*}{$\begin{array}{l}\text { Rinden- } \\
\text { extrakt }\end{array}$} & \multirow{2}{*}{$\begin{array}{l}\text { Paraform- } \\
\text { aldehyd } \\
\% \text { bez. auf } \\
\text { Klebstoff }\end{array}$} & \multicolumn{2}{|c|}{ Bindefestigkejt ${ }^{a}, \mathrm{~N} / \mathrm{mm}^{2}$} \\
\hline & & $\begin{array}{l}\text { Trockenbinde- } \\
\text { festigkeit }\end{array}$ & $\begin{array}{l}\text { NaBbinde- } \\
\text { festigkeit }\end{array}$ \\
\hline I & - & 3.1 & 2,0 \\
\hline I & 0.5 & 3,3 & 2.3 \\
\hline II & - & 2,4 & Keine Festigkei \\
\hline II & 0,5 & 3,2 & 1,9 \\
\hline III & - & 3,0 & Keine Festigkei \\
\hline III & 0,5 & 3.4 & 2,2 \\
\hline IV & - & 2,3 & 1,1 \\
\hline IV & 0,5 & 2,9 & 2,0 \\
\hline V & - & 2,6 & 0,9 \\
\hline $\mathrm{V}$ & 0,5 & 3,2 & 2.8 \\
\hline
\end{tabular}

\footnotetext{
a Mittelwert von 10 Proben

b Anzahl der Proben ohne Festigkeit. hierbei ist der Mittelwert von den restlichen Proben angegeben
}

\begin{tabular}{llr}
\hline Extrakt & Rinde & Extraktionsmiltel (\% bez. auf atro Rinde) \\
\hline I & Fichte & $1 \% \mathrm{NaOH} / 2 \% \mathrm{Na}_{2} \mathrm{SO}_{3}$ in $\mathrm{H}_{2} \mathrm{O}$ \\
II & Fichte & $10 \% \mathrm{NaOH} / 2 \% \mathrm{Na}_{2} \mathrm{SO}_{3}$ in $\mathrm{H}_{2} \mathrm{O}$ \\
III & Kiefer & $7 \% \mathrm{NaOH} ;$ Extrakt unter schwach sauren \\
& & Bedingungen mit $2 \%$ Phenol (bez. auf \\
& & Feststofn modifiziert \\
IV & Kiefer & $5 \% \mathrm{NaOH}$ in Ethanol/ $\mathrm{H}_{2} \mathrm{O}(6: 4)$ \\
V & Fichte & $2 \% \mathrm{NaOH}$ in Ethanol $/ \mathrm{H}_{2} \mathrm{O}(6: 4)$ \\
\hline
\end{tabular}

Resultate: 1. Die mit Kombinationen aus einem nichtemulgierten Diisocyanat und Rindenextraktlösungen verleimten Sperrholzproben hatten nur bei Einsatz von Extrakt I Naßbindefestigkeit. 2. Die Zugabe von Paraformaldehyd zu den Klebergemischen erhöhte die Bindefestigkeiten der Sperrholzproben deutlich; Naßbindefestigkeiten wurden bei allen Verleimungen erzielt. - WKI-Kurzbericht Nr. 25/1986

\section{Mikroschnitte im Übergang zwischen Tangential- und Querschnitt in Nadelhölzern}

H. Schulz, Institut für Holzforschung der Universität München, Winzererstr. 45, 8000 München 40

Subject: Microcuts of different softwoods produced at an angle between tangential and cross section (influence: from tangential section $=70^{\circ}$, from cross section $=20^{\circ}$ ) showed in many places undulatory or zigzag patterns of tracheids in tangential direction (Fig.).

Mikroschnitte von Holz werden üblicherweise quer, tangential und/oder radial hergestellt und in diesen Schnittebenen unter dem Mikroskop beobachtet, beschrieben und ausgewertet. Es stellt sich die Frage, ob bei bestimmten Problemstellungen Übergangsschnitte zwischen diesen Ebenen für zusätzliche Einblicke nützlich sein können. Dies kann z. B. der Fall sein, wenn man etwas mehr "Tiefenschärfe" erreichen, also von einer Beobachtungsebene in die nächste eindringen will.

Material und Methode: 3 Proben aus verschiedenen Fichtenstämmen (Picea abies (L.) Karst.) sowie Einzelproben von Tanne (Abies alba Mill.), Kiefer (Pinus sylvestris L.), Strobe (Pinus strobus L.), Douglasie (Pseudotsuga menziesii (Mirb.) Franco), Lärche (Larix decidua Mill.) und Eibe (Taxus baccata L.).

Herstellung von Mikroschnitten auf dem Mikrotom im Übergang zwischen Tangential- und Querschnitt (um 20) aus der Tangentialebene in Richtung des Querschnitts abgewinkelt).

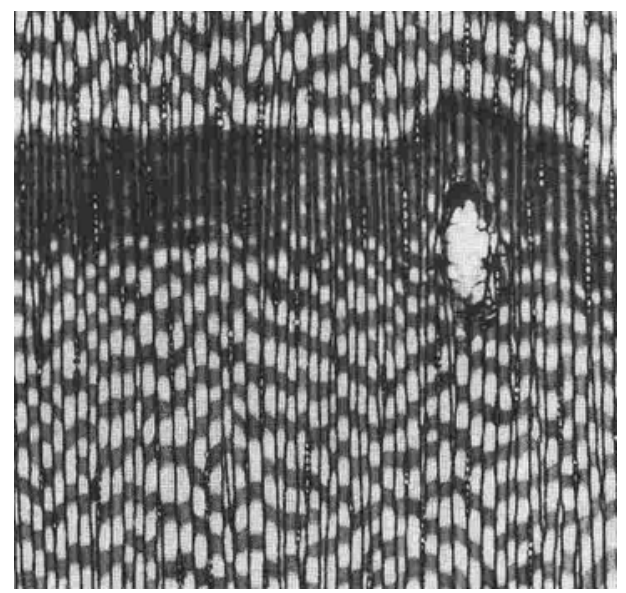

Resultate: 1. In Mikroschnitten von Fichte und den anderen genannten Nadelhölzern zeigten sich an vielen Stellen wellen- oder zickzackförmige Anordnungen der Tracheiden in tangentialer Richtung (Bild). 2. In den Fichtenschnitten und den Einzelschnitten von Tanne, Kiefer, Douglasie und Eibe war diese Erscheinung deutlicher ausgeprägt als in den Einzelschnitten von Strobe und Lärche. 3. Damit erhebt sich die Frage, ob neben der bekannten, von den einzelnen Kambiumzellen bestimmten radialen Ordnung der Tracheiden im Nadelholz auch in tangentialer Richtung gewisse Ordnungsprinzipien bestehen und in welchem Umfang Holzeigenschaften dadurch beeinflußt werden können. 